\title{
Definition of design guidelines, construction, and performance of an ultra- stable scanning tunneling microscope for spectroscopic imaging
}

Cite as: Rev. Sci. Instrum. 89, 123705 (2018); https://doi.org/10.1063/1.5064442

Submitted: 04 October 2018 . Accepted: 19 November 2018 . Published Online: 12 December 2018

Irene Battisti, Gijsbert Verdoes, Kees van Oosten, Koen M. Bastiaans (D), and Milan P. Allan (iD)

\section{ARTICLES YOU MAY BE INTERESTED IN}

Amplifier for scanning tunneling microscopy at $\mathrm{MHz}$ frequencies

Review of Scientific Instruments 89, 093709 (2018); https://doi.org/10.1063/1.5043267

Vibration isolation with high thermal conductance for a cryogen-free dilution refrigerator

Review of Scientific Instruments 90, 015112 (2019); https://doi.org/10.1063/1.5066618

Atomic scale shot-noise using cryogenic MHz circuitry

Review of Scientific Instruments 89, 093708 (2018); https://doi.org/10.1063/1.5043261 


\title{
Definition of design guidelines, construction, and performance of an ultra-stable scanning tunneling microscope for spectroscopic imaging
}

\author{
Irene Battisti, ${ }^{1}$ Gijsbert Verdoes, ${ }^{1,2}$ Kees van Oosten, ${ }^{1,2}$ Koen M. Bastiaans, ${ }^{1}$ \\ and Milan P. Allan ${ }^{1, a)}$ \\ ${ }^{1}$ Leiden Institute of Physics, Leiden University, 2333 CA Leiden, The Netherlands \\ ${ }^{2}$ Fine Mechanical Department, Leiden University, 2333 CA Leiden, The Netherlands
}

(Received 4 October 2018; accepted 19 November 2018; published online 12 December 2018)

\begin{abstract}
Spectroscopic-imaging scanning tunneling microscopy is a powerful technique to study quantum materials, with the ability to provide information about the local electronic structure with subatomic resolution. However, as most spectroscopic measurements are conducted without feedback to the tip, it is extremely sensitive to vibrations coming from the environment. This requires the use of laboratories with low-vibration facilities combined with a very rigid microscope construction. In this article, we report on the design and fabrication of an ultra-stable scanning tunneling microscope (STM) for spectroscopic-imaging measurements that operates in ultra-high vacuum and at low temperatures $(4 \mathrm{~K})$. We start from existing designs with sapphire as the main material and improve the stiffness further by performing finite element analysis calculations for the main components of the microscope to guide design choices on the geometry of the parts. With this strategy, we construct a STM head with measured lowest resonant frequencies above $f_{0}=13 \mathrm{kHz}$ for the coarse approach mechanism, a value three times higher than what has been previously reported and in good agreement with the calculations. This allows us to achieve an average vibration level of $\sim 6 \mathrm{fm} / \sqrt{\mathrm{Hz}}$, without a dedicated low-vibration lab. We demonstrate the microscope's performance with topographic and spectroscopic measurements on the correlated metal $\mathrm{Sr}_{2} \mathrm{RhO}_{4}$, showing the quasiparticle interference pattern in real and reciprocal space with high signal-to-noise ratio. Published by AIP Publishing. https://doi.org/10.1063/1.5064442
\end{abstract}

\section{INTRODUCTION}

Spectroscopic-imaging scanning tunneling microscopy (SI-STM) is an important tool to study the properties of quantum materials. ${ }^{1-3}$ The power of the technique stems from the ability of performing tunneling spectroscopy experiments with high energy and spatial resolution, allowing one to map the electronic local density of states (LDOS) onto a simultaneously acquired image of the atomic lattice. By observing quasiparticle interference (QPI), one can also access the reciprocal space, providing information about the band structure below and above the Fermi level. ${ }^{4-6}$ Since its application to the field of correlated electron systems and, in particular, to high$\mathrm{T}_{\mathrm{c}}$ superconductors, SI-STM allowed, among others, imaging the impact of individual dopant atoms on the electronic structure, ${ }^{7,8}$ visualizing nanoscale electronic disorder, ${ }^{9,10}$ imaging local symmetry breaking, ${ }^{11,12}$ and determining the gap structure by QPI imaging. 6,13

In order to achieve the data quality that is needed to understand the properties of correlated electron systems, there are some strict requirements for the design of the microscope. In a typical STM experiment, the tip scans over the sample to acquire a topographic image using a feedback mechanism that keeps the tunneling current constant by controlling the tip-sample distance. The feedback thus partially corrects

a)allan@ physics.leidenuniv.nl for vibrations transmitted to the tunnel junction, which are usually the main source of noise in STM. When performing a spectroscopy experiment, instead, the feedback loop is switched off to measure a $\mathrm{dI} / \mathrm{dV}$ spectrum, which is proportional to the LDOS. During this spectroscopic measurement, the instrument is very sensitive to external vibrations: Given the exponential dependence of the tunneling current on the tip-sample distance, even the smallest vibrations will make the tunneling current, and thus the $\mathrm{dI} / \mathrm{dV}$ spectrum, significantly noisier. In a typical SI-STM measurement, $10^{4}-10^{5}$ of such spectra distributed on a fine spacial grid are consecutively acquired over several days. This demands extreme stability of the microscope in order to achieve the signal-tonoise ratio $(\mathrm{S} / \mathrm{N})$ needed to study the properties of quantum materials.

Traditionally, high stability is achieved by combining the use of a vibration isolation table with a rigid microscope construction. Both are essential to obtain good data quality; however, during the design phase, it is mostly important to focus on the rigidity of the STM head, it being the factor that can drastically improve the performance of the microscope. ${ }^{14}$ This is the focus of the present paper.

A good way of quantifying the rigidity of the STM head is via its lowest resonant frequency $f_{0}$, usually in the $\mathrm{kHz}$ range. The reason for this can be seen in a simple harmonic oscillator model. It can be shown ${ }^{14}$ that, for $f \ll f_{0}$, the vibration transfer function from the environment to the tunneling junction 
is inversely proportional to $f_{0}^{2}$. Due to this quadratic dependence, even small improvements to $f_{0}$ would already reduce the vibration level at the junction by one order of magnitude. Because the current is exponential in distance, this leads to tremendous reductions in the current noise. A good criterion to achieve a high S/N is thus to build a STM head with the highest possible value of $f_{0}{ }^{14,15}$

In the following, we report on the design, construction, and performance of a home-built STM for spectroscopic imaging measurements, defining guidelines in order to obtain a very stable microscope. The instrument is ultra-high vacuum (UHV) compatible and operates at low temperatures (4 K). In Sec. II, we focus on the STM head design and construction, and we explain how we achieve a factor three higher stiffness than previously reported ${ }^{15}$ by using finite element analysis (FEA) to improve the geometry of all the components of the STM head. In Sec. III, we give an overview of the cryogenic insert and of the vibration isolation table. Finally, in Sec. IV, we show the performance of the STM and report vibrational noise spectra and high quality topographic and spectroscopic data measured at $4 \mathrm{~K}$ on the correlated metal $\mathrm{Sr}_{2} \mathrm{RhO}_{4}$.

\section{DESIGN AN ULTRA-STIFF STM HEAD USING FINITE ELEMENT ANALYSIS}

The stiffest cryogenic-compatible STM head that is reported in the literature to date is based on the design of Pan et al. ${ }^{16}$ using oriented single-crystalline sapphire as the material for the body of the STM head. ${ }^{15}$ We take this as our starting point. Here, in order to improve the stiffness even further, we optimize the geometry of all the components of the head by guiding the design choices with finite element analysis (FEA) calculations ${ }^{17}$ towards achieving a higher $f_{0}$ of the fully assembled STM head. FEA calculations have been used before to improve the geometry of various scanning probe techniques ${ }^{18-21}$ and, in particular, of high-scanning speed STM. ${ }^{22-24}$ Here, we use them to improve the geometry of a low-temperature STM for spectroscopic imaging. With this approach, we are able to additionally increase the stiffness of the head by a factor of three with respect to the work of White et al. ${ }^{15}$

\section{A. Design of the STM head}

In Fig. 1, a computer aided design (CAD) rendering and pictures of the assembled STM head are shown. The STM body is machined from single-oriented crystalline sapphire. ${ }^{25}$ Sapphire is an ideal material because it is one of the stiffest insulating materials available and it has an excellent low temperature thermal conductivity. ${ }^{15}$ Recently, it has become easier and more affordable to machine, allowing us to design more complicated and detailed parts for the STM head than what was previously possible.

The head consists of a hollow cylindrical sapphire body open on one side, inside which a triangular polished sapphire prism (the slider) is clamped via six shear piezo stacks ${ }^{26}$ and a molybdenum plate acting as the spring. The piezo stacks are glued with non-conductive epoxy ${ }^{27}$ to the sapphire body, while the triangular prism is just clamped and can slide up and down with respect to the body when high voltage pulses are applied to the piezo stacks. This whole construction provides the coarse approach mechanism, otherwise called walker, that allows the tip to move towards the sample over a $5 \mathrm{~mm}$ range with nanometer-sized steps (at $4 \mathrm{~K}$, with $120 \mathrm{~V}$ pulses, one step up is about $20 \mathrm{~nm}$ ). It is essential to the functioning of the walker to have very flat and perfectly parallel surfaces at the contact between the slider and the piezostacks, which requires polishing of the contacting surfaces.

The piezotube, ${ }^{28}$ providing the scanning motion both in the $\mathrm{Z}$ (vertical) and in the $\mathrm{XY}$ (in-plane) directions, is glued with non-conductive epoxy on top of a Macor support which is in turn fixed inside the slider. It has 4 outer electrical contacts for deflections in the $\pm \mathrm{X}, \pm \mathrm{Y}$ directions and one inner contact for contractions and elongation in Z. It is driven with maximum $\pm 100 \mathrm{~V}$ on all contacts, ${ }^{29}$ resulting at $4 \mathrm{~K}$ in a XY field of view of $380 \mathrm{~nm}^{2}$ and a $\mathrm{Z}$ elongation of $110 \mathrm{~nm}$.

The tip is mounted on a tip holder glued on top of the piezotube. The tip holder assembly is optimized for low weight, in order to increase the resonant frequencies of the (a)

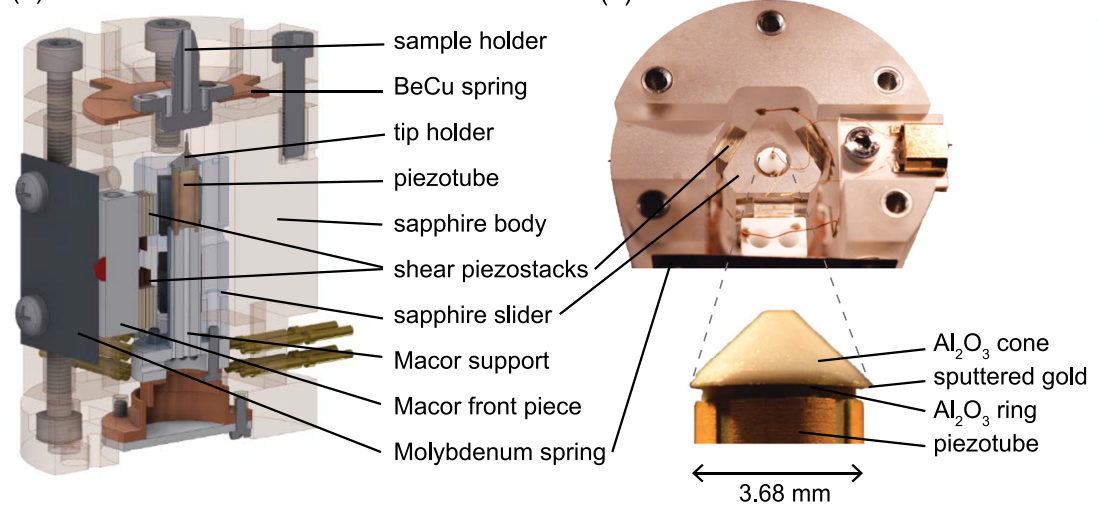

(c)

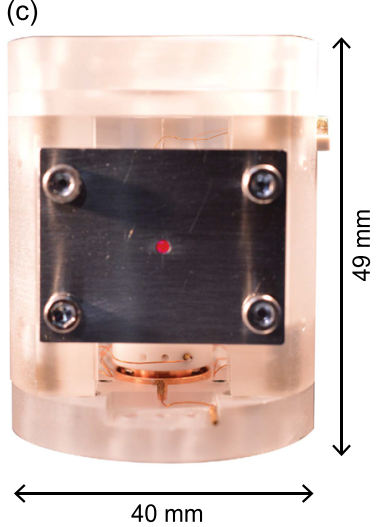

FIG. 1. (a) Cross section of the 3D CAD rendering of the STM head, with the main components being pointed out. (b) Top view of the open STM head, where the slider construction and the tip holder can be seen. The picture of the tip holder during the construction phase is shown in detail. The conical $\mathrm{Al}_{2} \mathrm{O}_{3}$ holder has been sputtered with gold on the bottom surface to provide a ground shield for the current signal (measured from the tip). In between the latter and the piezotube, a small $\mathrm{Al}_{2} \mathrm{O}_{3}$ ring is glued. (c) Picture of the fully assembled STM head. 
scanner, and for low capacitance, in order to allow for RFSTM measurements. ${ }^{30}$ It consists of a small $\mathrm{Al}_{2} \mathrm{O}_{3}$ ring glued directly on the piezotube and a conical $\mathrm{Al}_{2} \mathrm{O}_{3}$ holder glued on the ring, as shown in Fig. 1(b). This last piece hosts a small molybdenum cylinder inside which the tip can be clamped by friction. The bottom surface of the conical piece is additionally sputtered with $450 \mathrm{~nm}$ of gold to provide a ground shield for the tip wire. With this assembly, we obtain a tip-tosample capacitance of $180 \mathrm{fF}$ (at tunneling distance, with tip retracted) and a tip-to-ground shield capacitance of $2 \mathrm{pF}$. The tip-to-ground shield capacitance should be as low as possible to facilitate RF-STM measurements, and the value we measure is reduced by more than a factor of 10 with respect to commercially available instruments.

The sample holder receptacle is on top of the main body, with the sample facing downwards towards the tip and clamped by a BeCu spring. The scanner is not provided with an $\mathrm{XY}$ stage for coarse motion of the sample holder, in order to not reduce the stability of the assembly.

\section{B. Design principles from finite element analysis}

The optimization procedure that we applied to several components of the STM head in order to achieve high resonant frequencies is shown in Fig. 2(a). The FEA calculations are performed using the commercially available software Comsol. ${ }^{31}$ As an example, we show here how we apply this procedure to the piezotube. After importing the initial design in Comsol, we compute the mechanical eigenfrequencies of the first vibrational modes with the base of the piezotube fixed, as shown in Fig. 2(b). We can identify two double degenerate bending modes, a rotational mode and a vertical mode. We then progressively change the dimensions of the part and recompute the eigenfrequencies, in order to understand which dimensions can be optimized to increase the stability. In the case of a simple tube, this problem could also be solved analytically; however, here we want to show the procedure that we will apply to more complicated parts of the STM head. We find that is beneficial to lower the height [Fig. 2(c)], while changing the wall thickness (with fixed outer diameter) does not have a significant effect [Fig. 2(d)]. In general, the optimization towards higher resonant frequencies can be limited by functional requirements and geometrical constraints. For the piezotube, the final design is chosen as a compromise between stability and the desired XY scanning range of $\geq 350 \mathrm{~nm}$ at $4 \mathrm{~K}$.

A similar analysis is performed on many other STM head components, including the sapphire slider, the STM body, the Macor front piece, and the sample holder. For instance, the simulations indicate that to reach a higher $f_{0}$, the STM head will benefit from a lower aspect ratio, a shorter and lighter sapphire slider, and a lighter Macor front piece. The simulations also help determining until which point removing mass, for instance by drilling holes in the Macor front piece or in the sample holder, would help and when instead it would start hindering the stiffness of the part.

Importantly, we also performed FEA calculations on the whole assembly of the STM head, simplified by removing small details in the design, to allow for a simpler finite (a)

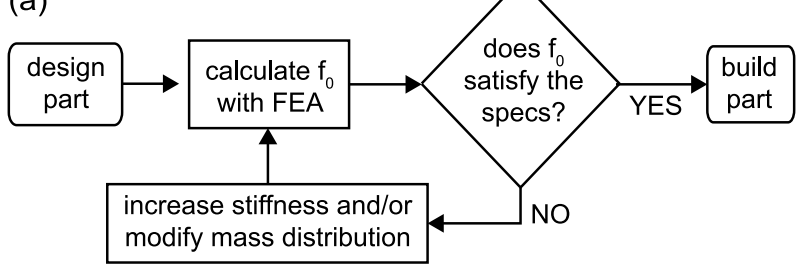

(b)
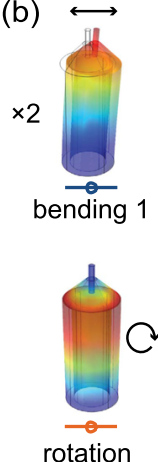

rotation

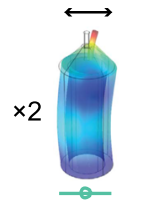

bending 2

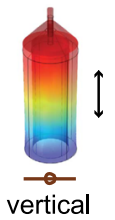

(c)

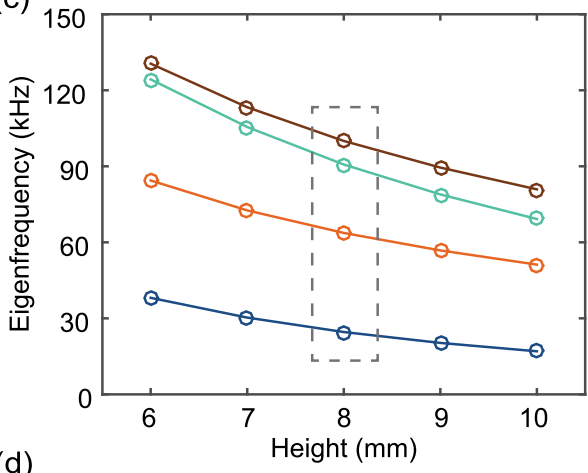

(d)

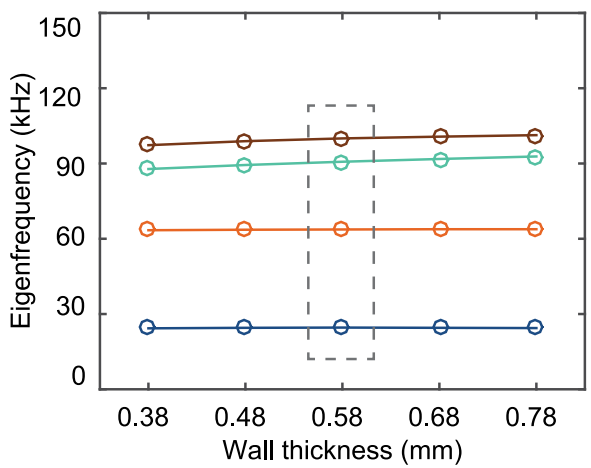

FIG. 2. (a) Flow chart explaining the procedure applied to several components of the STM head in order to achieve higher resonant frequencies. (b) Mode shapes of the first six mechanical resonances of the piezotube as computed with Comsol, as a legend for panels [(c) and (d)]. The modes are shown as (exaggerated) displacements with colors from red (max displacement) to blue (no displacement). (c) Variation of eigenfrequencies of the piezotube's modes with respect to the piezotube height, with base diameter fixed. (d) Variation of eigenfrequencies of the piezotube's modes with respect to the wall thickness (with fixed outer diameter $D=3.68 \mathrm{~mm}$ ). The dashed boxes indicate the eigenfrequencies for the final chosen dimensions.

element mesh. This can provide further insights about the design choices, showing which are the less stiff parts of the head where most attention has to be paid, and moreover it can be directly compared to the measured values of the eigenfrequencies. The most significant modes that we obtain from FEA calculations of the full STM head after optimization are shown in Fig. 3. They can be divided in two groups: the modes belonging to the body of the STM ( $b$-modes in Fig. 3 ) and the modes belonging to the scanner assembly ( $s$-modes). The modes $b_{1}, b_{2}$ are almost degenerate and correspond to the bending modes of the full head, $b_{3}$ corresponds to the rotational mode, and $b_{4}$ corresponds to the motion of the slider inside the head. The modes $s_{1}$ and $s_{2}$ are double degenerate and correspond to first order and second order bending mode of the scanner, $s_{3}$ is the rotational mode of the scanner, $s_{4}$ is the vertical mode of the scanner, and $s_{5}$ is the bending mode of the tip. 


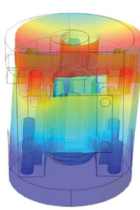

$\mathrm{b}_{1}=13.0 \mathrm{kHz}$

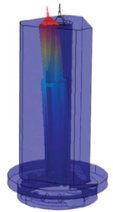

$\mathrm{s}_{1}=4.3 \mathrm{kHz}$

(x2)

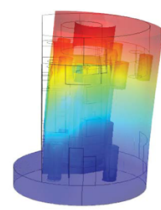

$b_{2}=13.5 \mathrm{kHz}$
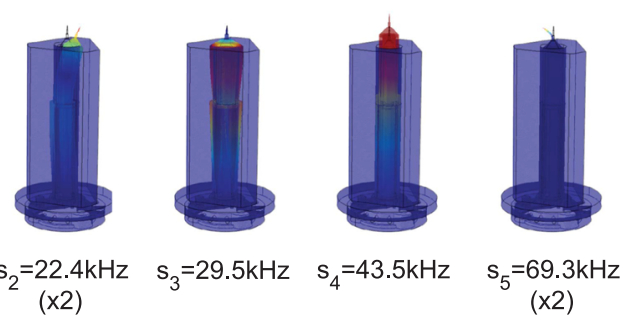

(x2)

$\mathrm{s}_{4}=43.5 \mathrm{kHz}$

$\mathrm{s}_{5}=69.3 \mathrm{kHz}$

(x2)

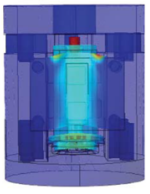

$\mathrm{b}_{4}=32.9 \mathrm{kHz}$
FIG. 3. Main vibrational modes of the STM head, as calculated with Comsol, with the base of the STM fixed. All the modes shown are calculated for the full design, but to ease visualization, the modes that belong to the scanner assembly $\left(s_{1}-s_{5}\right)$ are shown separately. See the main text, Sec. II B, for a description of the modes.

Intuitively, one could think that the walker assembly used for the coarse approach is the weakest point of the STM head design and, therefore, that the lowest vibrational mode should correspond to the vertical motion of the slider inside the scanner $\left(b_{4}\right)$. In our simulations, however, the main body vibrational modes and the scanner bending modes have lower frequencies, showing that the limiting factor for $f_{0}$ is mainly the STM head geometry. Clearly, the calculated value of the mode $b_{4}$ depends strongly on the stiffness of the shear piezo stacks that we feed into the simulation. The value of $b_{4}$ shown in Fig. 3 is obtained with half the stiffness reported in the specifications from the manufacturer, to take into account the effect of glue and other factors involved in the mounting (we first model each shear piezo stack as a single block of piezoelectric material, adjusting its Young's modulus to meet the stiffness value from the specifications, and then half the value of the Young's modulus was used as the input of the simulation). Furthermore, the measured resonant frequencies reported in Sec. II C confirm that the slider construction is not the weakest point of the design.

\section{Measurement of resonant frequencies}

After the construction of the STM head, we measure both the eigenfrequencies of the walker assembly and of the piezotube assembly independently and we compare them with the calculations. The measurement is performed in situ with the head mounted in the cryogenic insert at room temperature and under vacuum. A lock-in amplifier is used to excite the piezo elements and to read out their response. The applied excitation is a sinusoidal signal of amplitude $1 V_{\text {peak-peak }}$ and sweeping frequency. A scheme of the circuit is presented in the inset of Fig. 4(a). ${ }^{22}$ Thanks to the intrinsic properties of piezoelectric materials, the electrical excitations $V_{\text {in }}$ provided by the lock-in is converted into a mechanical excitation. When the excitation frequency corresponds to one of the STM eigenfrequencies, its amplitude gets enhanced, resulting in an enhanced electrical signal from the piezos $V_{\text {out }}$ that is detected by the lock-in. ${ }^{22,23}$
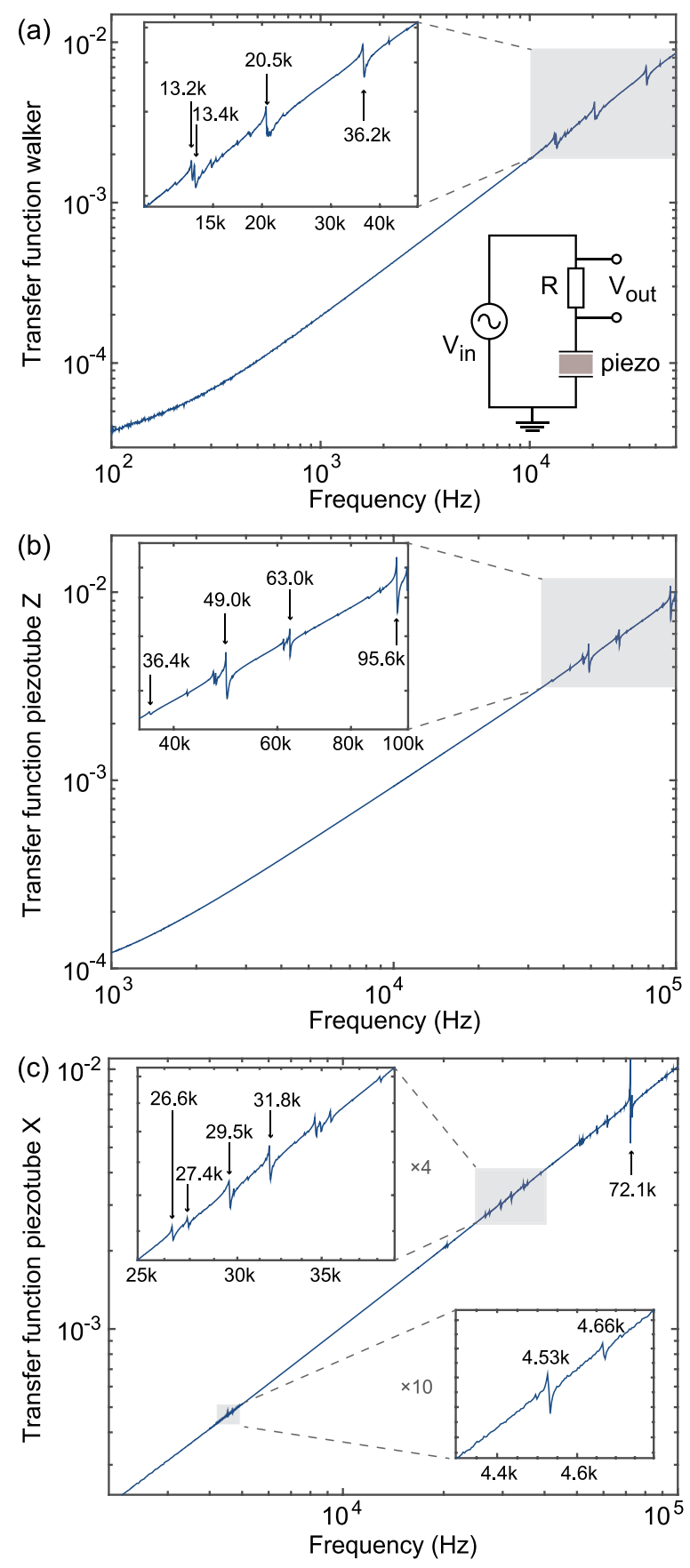

FIG. 4. Measured transfer functions $V_{\text {out }} / V_{\text {in }}$ of the coarse approach system and of the piezotube. (a) Transfer function of the shear piezo stack coarse approach mechanism. A scheme of the electronic circuit is shown at the bottom right corner, where $R=11 \Omega$. In the inset, a zoom of the area shaded in gray is shown. (b) Transfer function of the piezotube vertical excitation. In the inset, a zoom of the gray area is shown. (c) Transfer function of the piezotube bending excitation. In the insets, zooms of the gray areas are shown (note the different scales).

Figure 4(a) shows the transfer function obtained when exciting the six shear piezo stacks for the coarse approach connected in parallel. The main result is that we do not observe any resonant mode of the coarse approach assembly before $10 \mathrm{kHz}$, with the first strong peaks appearing at $\sim 13 \mathrm{kHz}$. We also performed the measurement at $4 \mathrm{~K}$ (not reported here), leading to slightly higher resonance frequencies due to the different stiffness of the materials at low temperatures and to 
thermal contractions. Remarkably, the value we achieve is a factor of three better than the previously reported eigenfrequencies for a sapphire STM head, ${ }^{15}$ where the first strong peak in the coarse approach excitation appears at $4.6 \mathrm{kHz}$.

Additionally, we can now compare the measured resonances with the computed ones. We can easily identify the first two almost degenerate peaks at $13.2 \mathrm{kHz}$ and $13.4 \mathrm{kHz}$ as the two bending modes of the STM body $\left(b_{1}, b_{2}\right)$. The peak at $20.5 \mathrm{kHz}$ is more difficult to identify, but it could correspond to the rotational mode $b_{3}$. We interpret the peak measured at $36.2 \mathrm{kHz}$ as the mode $b_{4}$, given that in the FEA calculations, $b_{4}$ is the only mode we find in the frequency window between $30 \mathrm{kHz}$ and $50 \mathrm{kHz}$ and in the measurement we do not observe any other peak before $\sim 80 \mathrm{kHz}$.

For the piezotube, we can measure the transfer function while exciting either the vertical modes (which are the most crucial ones) or the bending modes (which have less influence on the noise), simply by applying the excitation to different electrodes. In Fig. 4(b), we show the transfer function of the vertical modes, measured with the outer electrical contacts $( \pm \mathrm{X}, \pm \mathrm{Y})$ connected in parallel against the inner contact $(\mathrm{Z})$ acting as the ground. The small peak at $36.4 \mathrm{kHz}$ results from coupling to the mode $b_{4}$, and the peak at $49.5 \mathrm{kHz}$ can be identified as the vertical mode $s_{4}$ of the full scanner assembly. The peaks at $63.0 \mathrm{kHz}$ and at $95.6 \mathrm{kHz}$ are more difficult to identify, but could correspond to the rotational and vertical modes of the piezotube alone, which the calculations give, respectively, at $63.7 \mathrm{kHz}$ and $100.0 \mathrm{kHz}$ (from Fig. 2). In Fig. 4(c), we show the transfer function of the bending modes, measured between $+X$ and $-X$ contacts. The two very small peaks measured at $4.53 \mathrm{kHz}$ and $4.66 \mathrm{kHz}$ correspond to the first order bending modes of the piezotube $s_{1}$. The peaks at $26.6 \mathrm{kHz}$ and $27.4 \mathrm{kHz}$ could correspond to the second order bending modes $s_{2}$ and the peak at $29.5 \mathrm{kHz}$ to the rotational mode $s_{3}$. We do not find correspondence to the calculations for the other small peaks, and the strong peak at $72.1 \mathrm{kHz}$ could correspond to the bending mode of the tip $s_{5}$, even if we are doubtful that this could lead to such a sizable response of the piezotube.

The lowest resonant frequencies of the STM head thus correspond to the bending modes of the piezotube and of the STM head, which are substantially less critical for STM measurements than the vertical modes. The walker assembly, often a critical point for the vertical stability, proves to be the stiffest reported to date for a Pan-style head. Taken together, this confirms the exceptional stiffness of our STM head.

\section{DESIGN OF CRYOGENIC INSERT AND VIBRATION ISOLATION TABLE}

The STM head is mounted on the bottom of a home-built cryogenic insert placed in a ${ }^{4} \mathrm{He}$ bath cryostat and it operates at the base temperature of $4.3 \mathrm{~K}$. The insert is rigidly connected to the UHV chamber and the table, which is in turn suspended on vibration isolation springs standing on top of three legs. The full system is placed on a measurement island that is decoupled from the surrounding walking floor, but is not additionally isolated with dampers from the foundations. A picture of the cryogenic insert and a CAD rendering of the full system are shown in Fig. 5. (a)

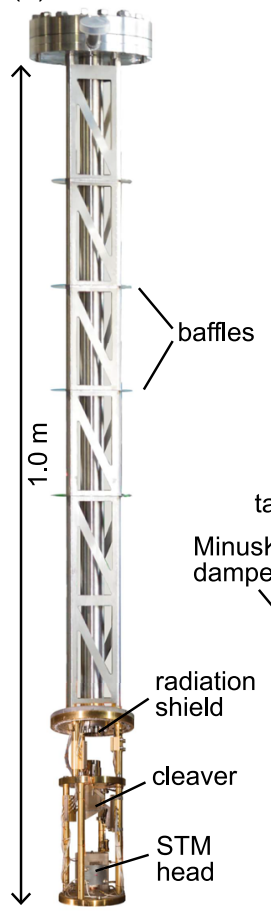

(b)

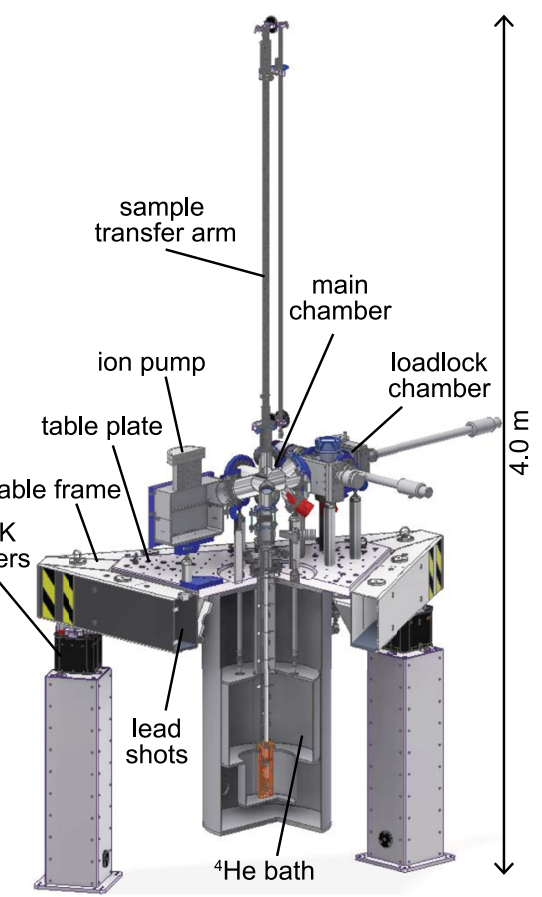

FIG. 5. (a) Picture of the cryogenic insert. (b) Three quarters section of the CAD rendering of the full system.

\section{A. Cryogenic insert}

The bottom part of the insert, where the STM is rigidly connected without the use of springs, is made of gold-plated oxygen-free copper to optimize the thermal connection to the ${ }^{4} \mathrm{He}$ bath. It is provided with a radiation shield and a cold cleaving stage that can be actuated by the sample transfer mechanism. The connection to the room temperature UHV chamber is made of thin-walled stainless steel tubes that are used for pumping and wiring.

The design of the cryogenic insert has also been optimized for maximum stability. For this reason, we opted for a reinforced insert structure that features four stainless steel tubes. The central tube has an outer diameter of $31.0 \mathrm{~mm}$ and a wall thickness of $0.5 \mathrm{~mm}$ and it is used as the sample transfer line and main pumping line. The three smaller tubes (of outer diameter $12.7 \mathrm{~mm}$ and wall thickness $0.25 \mathrm{~mm}$ ) are used for wiring and are placed in a triangular pattern around the central one. We find that choosing three tubes for wiring, instead of two, drastically improves the stability for the pendulum mode, with the resonant frequency corresponding to this mode increasing by $50 \%$. Furthermore, we provide additional stability by connecting the tubes with thin $(0.3 \mathrm{~mm})$ stainless steel plates. These plates have triangular holes in order to reduce the heat conductivity and are laser spot welded directly to the tubes. Around the tubes, and perpendicular to them, we place four stainless steel baffles that act as radiation shields inside the Dewar neck to reduce the radiation heat transfer to the ${ }^{4} \mathrm{He}$ bath.

All the extra features that we added to improve the stability imply a higher heat conduction to the ${ }^{4} \mathrm{He}$ bath, and a compromise has to be found between stability and desired helium consumption. With our particular design, we still obtain an 
excellent helium consumption of $0.18 \mathrm{l} / \mathrm{h}$ ( $4.3 \mathrm{l}$ /day), which ensures a very quiet helium boil-off noise and allows for $>7$ days' long measurements.

\section{B. Vibration isolation table}

The vibration isolation table is made of two parts: a rigid stainless steel hollow frame with a triangular shape and a $40-\mathrm{mm}$-thick triangular aluminum plate. The plate is rigidly connected to the frame by screws, and the assembly is designed with the specifications of not having resonant frequencies below $300 \mathrm{~Hz}$. Both the UHV chamber and the Dewar are rigidly fixed to the aluminum plate. In order to dampen acoustic-induced noise and vibrations and to increase the mass of the table, the frame is filled with lead shots to reach a weight of the total system of $\sim 1000 \mathrm{~kg}$. The table is suspended on three negative-stiffness vibration isolators, ${ }^{32}$ with resonant frequency $0.5 \mathrm{~Hz}$, that are mounted on three legs.

\section{PERFORMANCE}

\section{A. Vibrations and $I / z$ noise at the tunneling junction}

As a first test of the performance of our microscope, we measure the vibrational noise level at the tunneling junction.

We first characterize the noise level of our laboratory by measuring both vibrations with a seismometer (Guralp CMG-40T) and acoustic noise with a low-frequency microphone (G.R.A.S. 46AF). The measurement is reported as the linear spectral density (LSD) of the velocity for the vibrations and of the pressure difference for the acoustics. The spectra are obtained by Fourier transforming 1 min of real time data with the Welch method using a Hamming window.

We report in Fig. 6(a) typical spectra of (i) the vibrations measured on the island where the table is standing, (ii) the vibrations measured on the table, and (iii) the sound measured in the surrounding room. It can immediately be noticed that the table dampens the general vibrational noise by about one order of magnitude after $10 \mathrm{~Hz}$, and it reduces many of the peaks. The table is less effective in reducing the sound-induced noise, as it can be noticed from the peak at $24 \mathrm{~Hz}$ that is only partially dampened.

In Fig. 6(b), we show the vibrational noise at the tunnel junction as the linear spectral density of the $\mathrm{Z}$ height (corresponding to the tip-sample distance) measured in feedback on $(\mathrm{Pb}, \mathrm{Bi})_{2} \mathrm{Sr}_{2} \mathrm{CuO}_{6+x}$ at setup condition $\mathrm{V}=300 \mathrm{mV}$ and $\mathrm{I}=150 \mathrm{pA}$. Remarkably, the noise is very low, with an average vibration level of $\sim 6 \mathrm{fm} / \sqrt{\mathrm{Hz}}$ and only two peaks with amplitude bigger than $20 \mathrm{fm} / \sqrt{\mathrm{Hz}}$, respectively, at $12 \mathrm{~Hz}$ and $50 \mathrm{~Hz}$.

In Fig. 6(c), we further show the linear spectral density of the current noise measured with the same tunneling junction and setup condition. With the tip retracted, we achieve an average noise level of $\sim 8 \mathrm{fA} / \sqrt{\mathrm{Hz}}$. The preamplifier we use (FEMTO LCA-4K-1G) has a noise floor of $6.5 \mathrm{fA} / \sqrt{\mathrm{Hz}}$.

In tunneling and with the feedback loop closed, we can see an increase in the noise to $\sim 20 \mathrm{fA} / \sqrt{\mathrm{Hz}}$ above $200 \mathrm{~Hz}$, with the addition of some peaks reaching up to $\sim 50 \mathrm{fA} / \sqrt{\mathrm{Hz}}$. (a)

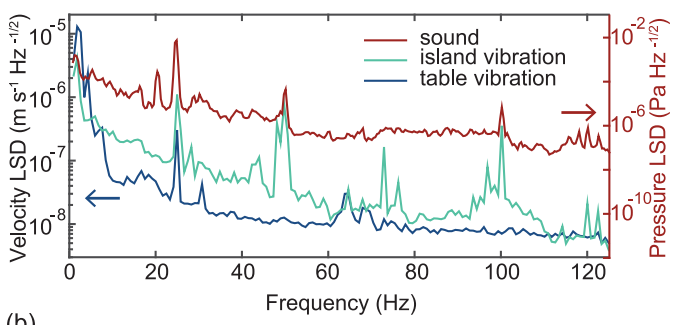

(b)

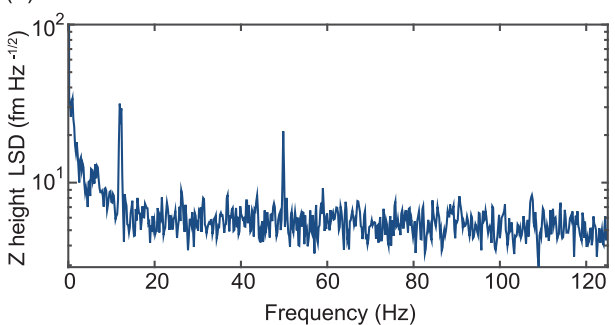

(c)

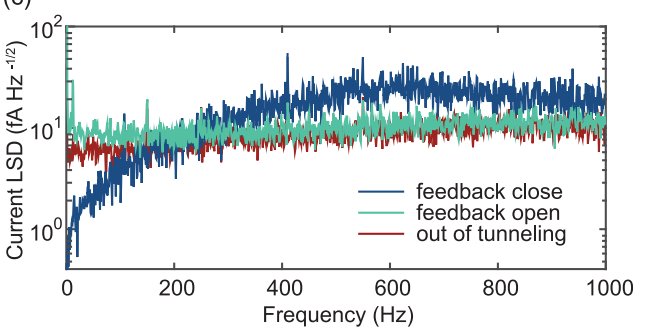

FIG. 6. (a) Linear spectral density (LSD) of the velocity measured in the Z direction on the island and on the STM table with a seismometer (Guralp CMG-40T). In the same graph, with the scale on the right axis, acoustic noise is reported as LSD of the pressure difference measured with a low-frequency microphone (G.R.A.S. 46AF). (b) LSD of the $\mathrm{Z}$ height noise at the tunnel junction measured on $(\mathrm{Pb}, \mathrm{Bi})_{2} \mathrm{Sr}_{2} \mathrm{CuO}_{6+x}$ at setup condition $\mathrm{V}=300 \mathrm{mV}$ and $\mathrm{I}=150 \mathrm{pA}$. (c) LSD of the tunneling current at the same tunnel junction as (b), measured out of tunneling as well as in tunneling with the feedback loop closed and open.

The feedback takes care of reducing the low frequency noise.

An important figure of merit for spectroscopic measurements is the out-of-feedback current noise (in tunneling and with the feedback loop open). We obtain a noise level of $\sim 10 \mathrm{fA} / \sqrt{\mathrm{Hz}}$, with only the peaks at $12 \mathrm{~Hz}$ and $50 \mathrm{~Hz}$ higher than $20 \mathrm{fA} / \sqrt{\mathrm{Hz}}$, which ensures the capability of performing high quality spectroscopy experiments.

\section{B. Topograph and QPI on $\mathrm{Sr}_{2} \mathrm{RhO}_{4}$}

To demonstrate the topographic and spectroscopic capabilities of the microscope, we show quasiparticle interference measurements on the correlated metal $\mathrm{Sr}_{2} \mathrm{RhO}_{4}$, which is known to have a highly two-dimensional electronic structure. ${ }^{33-35}$

The sample is cleaved at the $4 \mathrm{~K}$ cleaving stage and immediately transferred to the STM head. Measurements are performed with a chemically etched tungsten tip that has previously been prepared by field emission on a gold surface. Atomic resolution can be easily achieved, as shown in the $15 \times 15 \mathrm{~nm}^{2}$ topograph in Fig. 7(a) and in the linecut along the black line plotted in Fig. 7(b). The cleaved surface shows the $\mathrm{SrO}$ layer, with the distance between the $\mathrm{Sr}$ atoms being $3.9 \AA$, and two different defects. ${ }^{36}$ In Fig. 7(c), we plot a 

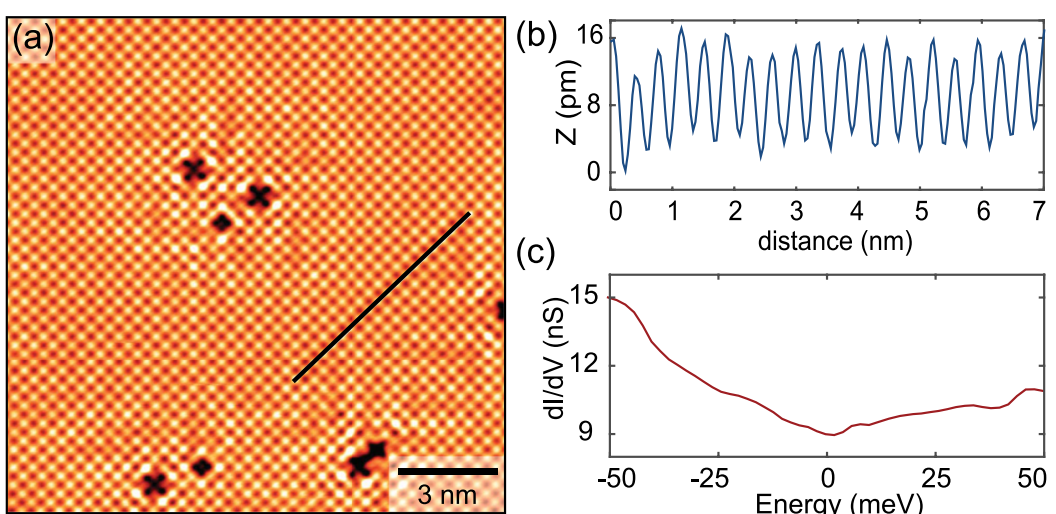

(c)
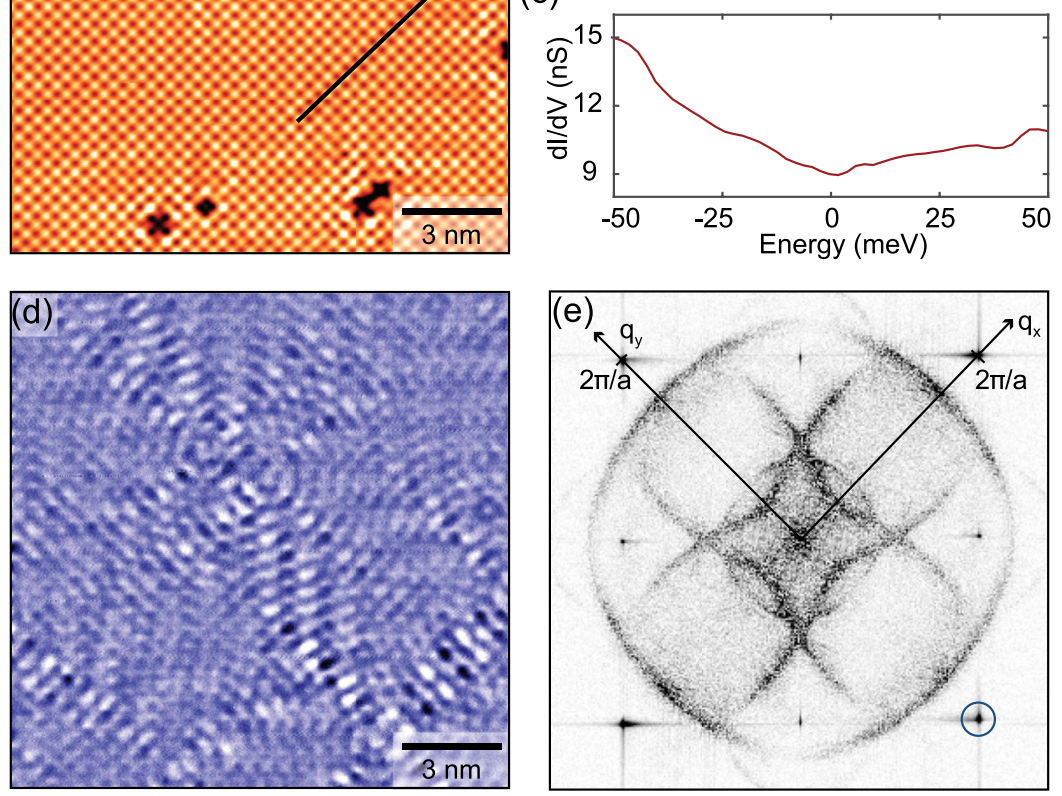

FIG. 7. STM measurements on $\mathrm{Sr}_{2} \mathrm{RhO}_{4}$. (a) Topograph in a field of view of $15 \times 15 \mathrm{~nm}^{2}$. Setup condition $\mathrm{V}=-20 \mathrm{mV}, \mathrm{I}=600 \mathrm{pA}$. The image is not filtered. (b) Atomic corrugation profile along the black line in (a). (c) Example of a single $\mathrm{dI} / \mathrm{dV}$ spectrum measured during a spectroscopic map. (d) Density of states measured simultaneously to the topograph in panel (a), showing the quasiparticle interference pattern in real space at energy $E=-20 \mathrm{meV}$. (e) Non-symmetrized Fourier transform of the conductance layer at the Fermi level acquired in a spectroscopic map over a $55 \times 55 \mathrm{~nm}^{2}$ field of view. One of the Bragg peaks is highlighted in the blue circle. simple dI/dV spectrum acquired during a spectroscopic map. In Fig. 7(d), we show the real space imaging of QPI in the conductance layer at $-20 \mathrm{meV}$ (with respect to the Fermi level), acquired simultaneously to the topograph in panel (a). The defects obviously act as scattering centers, creating an interference pattern that enhances the quantum mechanical wave nature of the quasiparticles. In Fig. 7(e), we show the Fourier transform of the real-space QPI pattern, ${ }^{35}$ measured at the Fermi level when taking a spectroscopic map on a field of view of $55 \times 55 \mathrm{~nm}^{2}$. The Fourier-transformed data is not symmetrized nor processed in any other way and still shows sharp features with very high signal-to-noise ratio.

A more comprehensive analysis, including a quantitative comparison with angle-resolved photoemission spectroscopy and quantum oscillation data, ${ }^{33,34}$ will be published elsewhere.

\section{v. CONCLUSIONS}

In this paper, we report on improved design principles, construction, and performance of an ultra-stable STM for spectroscopic imaging. We show that by improving the geometry of existing designs guided by finite element analysis calculations, we are able to improve the stiffness of the STM head by a factor of three with respect to the previous designs, allowing us to significantly reduce the transmission of vibrational noise at the junction. We demonstrate the extreme stability of our instrument by obtaining an out-of-feedback current noise level of $\sim 10 \mathrm{fA} / \sqrt{\mathrm{Hz}}$. Moreover, our data on the correlated metal $\mathrm{Sr}_{2} \mathrm{RhO}_{4}$ prove the excellent performance of the microscope in the acquisition of spectroscopic measurements.

\section{ACKNOWLEDGMENTS}

We thank M. Rost, F. Galli, K. Heeck, M. Leeuwenhoek, T.-M. Chuang, P. Wahl, M. Hamidian, I. Firmo, and J. C. Davis for valuable discussions, T. van Klingeren, M. Witlox, E. Wiegers, D. Oosterlee, A. Geluk, R. Koehler, L. Crama, and W. van der Geest for technical support, and A. Tamai, F. Baumberger, and R. Perry for suggesting STM investigations on the rhodate samples and providing the crystals and unpublished data.

This work was supported by the Netherlands Organization for Scientific Research (NWO/OCW) as part of the Frontiers of Nanoscience (NanoFront) program and the VIDI talent scheme (Project No. 680-47-536).

\footnotetext{
${ }^{1}$ K. Fujita, M. Hamidian, I. Firmo, S. Mukhopadhyay, C. K. Kim, H. Eisaki, S.-I. Uchida, and J. C. Davis, Spectroscopic Imaging STM: Atomic-Scale Visualization of Electronic Structure and Symmetry in Underdoped Cuprates (Springer, 2012), Vol. 171, pp. 73-109.

${ }^{2}$ J. Lee, J. A. Slezak, and J. C. Davis, J. Phys. Chem. Solids 66, 1370 (2005). ${ }^{3}$ A. Yazdani, E. H. da Silva Neto, and P. Aynajian, Annu. Rev. Condens. Matter Phys. 7, 11 (2016).

${ }^{4}$ L. Petersen, P. Sprunger, P. Hofmann, E. Lægsgaard, B. Briner, M. Doering, H. Rust, A. Bradshaw, F. Besenbacher, and E. Plummer, Phys. Rev. B 57, R6858 (1998).

${ }^{5}$ M. Crommie, C. Lutz, and D. Eigler, Nature 363, 524 (1993).

${ }^{6}$ J. E. Hoffman, K. McElroy, D.-H. Lee, K. M. Lang, H. Eisaki, S. Uchida, and J. C. Davis, Science 297, 1148 (2002).

${ }^{7}$ S. H. Pan, E. W. Hudson, K. M. Lang, H. Eisaki, S. Uchida, and J. C. Davis, Nature 403, 746 (2000).

${ }^{8}$ E. W. Hudson, K. M. Lang, V. Madhavan, S. H. Pan, H. Eisaki, S. Uchida, and J. C. Davis, Nature 411, 920 (2001).

${ }^{9}$ K. M. Lang, V. Madhavan, J. E. Hoffman, E. W. Hudson, H. Eisaki, S. Uchida, and J. C. Davis, Nature 415, 412 (2002).

${ }^{10}$ K. McElroy, J. Lee, J. A. Slezak, D.-H. Lee, H. Eisaki, S. Uchida, and J. C. Davis, Science 309, 1048 (2005).
} 
${ }^{11}$ Y. Kohsaka, C. Taylor, K. Fujita, A. Schmidt, C. Lupien, T. Hanaguri, M. Azuma, M. Takano, H. Eisaki, H. Takagi, S. Uchida, and J. C. Davis, Science 315, 1380 (2007).

${ }^{12}$ M. J. Lawler, K. Fujita, J. Lee, A. R. Schmidt, Y. Kohsaka, C. K. Kim, H. Eisaki, S. Uchida, J. C. Davis, J. P. Sethna, and E.-A. Kim, Nature 466, 347 (2010).

${ }^{13}$ M. P. Allan, A. W. Rost, A. P. Mackenzie, Y. Xie, J. C. Davis, K. Kihou, C. H. Lee, A. Iyo, H. Eisaki, and T.-M. Chuang, Science 336, 563 (2012).

${ }^{14}$ C. J. Chen, Introduction to Scanning Tunneling Microscopy (Oxford University Press, 2000).

${ }^{15}$ S. C. White, U. R. Singh, and P. Wahl, Rev. Sci. Instrum. 82, 113708 (2011).

${ }^{16}$ S. H. Pan, E. W. Hudson, and J. C. Davis, Rev. Sci. Instrum. 70, 1459 (1999).

${ }^{17}$ Performed with the commercially available software Comsol, COMSOL Multiphysics version 5.2, 2015.

${ }^{18}$ J. H. Kindt, G. E. Fantner, J. A. Cutroni, and P. K. Hansma, Ultramicroscopy 100, 259 (2004).

${ }^{19}$ C. R. Ast, M. Assig, A. Ast, and K. Kern, Rev. Sci. Instrum. 79, 093704 (2008).

${ }^{20}$ J. Maess, A. J. Fleming, and F. Allgöwer, Rev. Sci. Instrum. 79, 015105 (2008).

${ }^{21}$ C. Braunsmann and T. E. Schäffer, Nanotechnology 21, 225705 (2010).

${ }^{22}$ M. J. Rost, L. Crama, P. Schakel, E. van Tol, G. B. E. M. van VelzenWilliams, C. F. Overgauw, H. ter Horst, H. Dekker, B. Okhuijsen, M. Seynen, A. Vijftigschild, P. Han, A. J. Katan, K. Schoots, R. Schumm, W. van Loo, T. H. Oosterkamp, and J. W. M. Frenken, Rev. Sci. Instrum. 76, 053710 (2005).
${ }^{23}$ M. J. Rost, G. J. van Baarle, A. J. Katan, W. M. van Spengen, P. Schakel, W. A. van Loo, T. H. Oosterkamp, and J. W. Frenken, Asian J. Control 11, 110 (2009).

${ }^{24}$ Y. I. Yanson, F. Schenkel, and M. J. Rost, Rev. Sci. Instrum. 84, 023702 (2013).

${ }^{25}$ The sapphire parts are machined by ultrasonic grinding with diamond tools.

${ }^{26} \mathrm{P}-121.01 \mathrm{~T}$ from PI Ceramics, customized with height $2.7 \mathrm{~mm}$, and polished $\mathrm{Al}_{2} \mathrm{O}_{3}$ end plates.

${ }^{27}$ Epotek H74F.

${ }^{28}$ EBL4 from EBL Products. Height $8.0 \mathrm{~mm}$, OD $3.68 \mathrm{~mm}$, wall thickness $0.58 \mathrm{~mm}$.

${ }^{29}$ Electronic controller Nanonis RC5 from Specs GmbH.

${ }^{30}$ K. M. Bastiaans, T. Benschop, D. Chatzopoulos, D. H. Cho, Q. Dong, Y. Jin, and M. P. Allan, Rev. Sci. Instrum. 89, 093709 (2018).

${ }^{31}$ COMSOL AB, Comsol multiphysics 5.2, 2015.

${ }^{32}$ Minus K® 800CM-1.

${ }^{33}$ R. S. Perry, F. Baumberger, L. Balicas, N. Kikugawa, N. J. Ingle, A. Rost, J. F. Mercure, Y. Maeno, Z. X. Shen, and A. P. Mackenzie, New J. Phys. 8, 175 (2006).

${ }^{34}$ F. Baumberger, N. J. Ingle, W. Meevasana, K. M. Shen, D. H. Lu, R. S. Perry, A. P. MacKenzie, Z. Hussain, D. J. Singh, and Z. X. Shen, Phys. Rev. Lett. 96, 246402 (2006).

${ }^{35} \mathrm{~T}$. Craig, Imaging Interacting Electrons in $\mathrm{Sr}_{2} \mathrm{RhO}_{4}$, M.S. thesis, University of St. Andrews, 2012.

${ }^{36}$ B. Stöger, M. Hieckel, F. Mittendorfer, Z. Wang, D. Fobes, J. Peng, Z. Mao, M. Schmid, J. Redinger, and U. Diebold, Phys. Rev. Lett. 113, 116101 (2014). 